\title{
Investigation of InGaN-based red/green micro-light-emitting diodes
}

\author{
ZHE ZHUANG, DAISUKE IIDA, KAZUHIRO OHKAWA*
}

Computer, Electrical and Mathematical Sciences and Engineering (CEMSE) Division, King Abdullah University of Science and Technology

(KAUST), Thuwal, 23955-6900, Saudi Arabia

*kazuhiro.ohkawa@kaust.edu.sa

Received XX Month XXXX; revised XX Month, XXXX; accepted XX Month XXXX; posted XX Month XXXX (Doc. ID XXXXX); published XX Month XXXX

\begin{abstract}
We investigated the performance of InGaN-based red/green micro-light-emitting diodes ( $\mu$ LEDs) ranging from $98 \times 98$ to $17 \times 17 \mu \mathrm{m}^{2}$. The average forward voltage at $10 \mathrm{~A} / \mathrm{cm}^{2}$ was independent of the dimension of $\mu$ LEDs. Red $\mu$ LEDs exhibited a larger blue-shift of the peak wavelength $(\sim 35 \mathrm{~nm})$ and broader full-width at half maximum $(\geq 50 \mathrm{~nm})$ at 2 to $50 \mathrm{~A} / \mathrm{cm}^{2}$ compared to green $\mu$ LEDs. We demonstrated that $47 \times 47 \mu \mathrm{m}^{2}$ red $\mu$ LEDs had an on-wafer external quantum efficiency of $0.36 \%$ at the peak wavelength of $626 \mathrm{~nm}$, close to the red primary color defined in the recommendation 2020 standard.
\end{abstract}

Micro-light-emitting diodes ( $\mu$ LEDs) have recently gathered considerable interest for next-generation display applications, such as smartphones and watches, augmented reality (AR), and virtual reality (VR) devices $[1,2]$. The primary colors (red, green, and blue, RGB) are required to be achieved separately to develop full-color displays. InGaN-based blue $\mu$ LEDs are the best choice for blue color displays because they have demonstrated remarkable performances, even when the device dimension decreases to $10 \mu \mathrm{m}$ or less $[3,4]$.

Two approaches could realize green and red colors. A simple method uses blue or violet $\mu$ LEDs to excite color converters, such as phosphors or quantum dots $[5,6]$. This method can easily integrate RGB colors into matrices on the same substrate [7], yet the colorconversion efficiency of hybrid devices must be improved [8]. Another approach chooses to fabricate red and green $\mu$ LEDs using different materials, AlInGaP for red $\mu$ LEDs [9] and InGaN for green $\mu$ LEDs [10]. This material difference causes a mismatched angular distribution between the red and green $\mu$ LEDs [11], leading to a noticeable color shift at different observed angles. Besides, AlGaInP red $\mu$ LEDs typically suffer from great efficiency reduction because of the high surface recombination when the device dimensions shrink [12].

Therefore, expanding interest was spent on developing InGaNbased red $\mu$ LEDs. The major challenge for achieving InGaN-based red $\mu$ LEDs is the significant reduction of the external quantum efficiency (EQE) owing to high-indium-content in the active region. The large lattice mismatch between the $\mathrm{InGaN}$ active region and $\mathrm{GaN}$ cladding layers results in many defects in InGaN quantum wells. To reduce the lattice mismatch, partly relaxed InGaN pseudo-substrates were utilized in $50 \times 50 \mu \mathrm{m}^{2}$ sized red InGaN $\mu$ LEDs, which were demonstrated with an on-wafer EQE (the output power was measured on a wafer instead of in the integrating sphere) of $0.09 \%$ at a current density of $40 \mathrm{~A} / \mathrm{cm}^{2}$ with a peak emission wavelength of $616 \mathrm{~nm}$ [13]. A recent $6 \times 6 \mu \mathrm{m}^{2} \mathrm{InGaN}$ red $\mu \mathrm{LED}$ was realized on porous $\mathrm{GaN}$ pseudo-substrates, and it achieved a record on-wafer EQE of $0.2 \%$ at a current density of $10 \mathrm{~A} / \mathrm{cm}^{2}$ with a peak emission wavelength of $632 \mathrm{~nm}$ [14]. To our knowledge, this on-wafer EQE is the highest reported value for InGaN red $\mu$ LEDs. Recently, a high wall-plug efficiency (WPE) of $16.8 \%$ at $621 \mathrm{~nm}$ was obtained at 0.8 $\mathrm{A} / \mathrm{cm}^{2}$ for $1 \times 1 \mathrm{~mm}^{2}$ InGaN-based LEDs [15]. This WPE value was the highest for standard InGaN red LEDs. Our group first developed a high-temperature growth technique for high-indium-content InGaN layers $[16,17]$, and then employed strain engineering strategies from
$\mathrm{Ga}_{2} \mathrm{O}_{3}$ substrates [18] and $\mathrm{GaN}$ templates [19] to active region structures [20]. We also improved the electrical and optical properties of InGaN LEDs in device design and fabrication [21,22]. Since InGaN-based green and red $\mu$ LEDs do not perform as well as blue $\mu$ LEDs, investigation of green and red $\mu$ LEDs is crucial to identify the direction for future improvements.

This work examined the performances of InGaN-based red/green $\mu$ LEDs ranging from $98 \times 98$ to $17 \times 17 \mu \mathrm{m}^{2}$. Currentvoltage $(I-V)$ curves were used to estimate the forward voltages and reverse leakage currents. We investigated the EL characteristics of red/green $\mu$ LEDs at different current densities, including the EL peak wavelength, full-width at half maximum (FWHM), on-wafer EQE and color gamut. Finally, we also evaluated the temperature dependence of EL emission for red/green $\mu$ LEDs.

InGaN-based red LED epitaxial wafers were grown on $c$-plane patterned sapphire substrates by metalorganic vapor-phase epitaxy (MOVPE) in a single-wafer horizontal reactor at $100 \mathrm{kPa}$ [16]. The epitaxial structures of the InGaN-based red LEDs were reported in our previous work [19]. Indium tin oxide (ITO) films were deposited as a transparent conductive layer and subjected to two-step annealing to form ohmic contacts with $\mathrm{p}-\mathrm{GaN}$ [21]. The $\mu \mathrm{LED}$ mesas ranging from $98 \times 98$ to $17 \times 17 \mu \mathrm{m}^{2}$ were patterned by standard photolithography and then etched through the ITO layer and InGaN active region by inductively coupled plasma (ICP) to expose the ntype layer. $\mathrm{A} \mathrm{SiO}_{2}$ isolated layer was then deposited using plasmaenhanced chemical vapor deposition (PECVD). Finally, we opened the $\mathrm{SiO}_{2}$ windows on the ITO and n-type layers by ICP etching and deposited $\mathrm{Cr} / \mathrm{Pt} / \mathrm{Au}(50 \mathrm{~nm} / 200 \mathrm{~nm} / 200 \mathrm{~nm})$ as n-type and p-type contact pads. All the red $\mu$ LEDs with different dimensions were fabricated on the same wafer. In the case of InGaN-based green $\mu$ LEDs, we used commercial epitaxial wafers on $c$-plane patterned sapphire substrates and performed the same fabrication process.

The electrical pumping of red and green $\mu$ LEDs was carried out at a probe station using a semiconductor parameter analyzer. The EL characteristics were measured under direct current operation ranging from $295 \mathrm{~K}$ (room temperature, RT) to $373 \mathrm{~K}$. The temperatures were measured from the sample-holding stage of the probe station. We also calculated the on-wafer EQE by the output power collected from the top side of the $\mu$ LEDs.

Figure 1(a) shows the absolute current density-voltage $(|J|-V)$ curves for InGaN-based red $\mu$ LEDs with the dimension from $98 \times 98$ to $17 \times 17 \mu \mathrm{m}^{2}$. The absolute current density was on a logarithmic 
scale. At the forward voltage, the $|J|-V$ curves were identical for all red $\mu$ LEDs with different dimensions. The turn-on voltage, which was regarded as the transition point of the two linear parts in the semi-logarithmic scale in Figure 1(a), was between 2.5 and $3 \mathrm{~V}$. However, at the reverse voltage, the absolute current density increased with decreasing the dimension of red $\mu$ LEDs. This behavior was opposite from our previous work [22] for red LEDs in different chip sizes larger than $100 \times 100 \mu^{2}$. We attributed this larger reverse leakage current for $17 \times 17 \mu \mathrm{m}^{2} \mu$ LEDs to the sidewall damages after ICP etching. Previous work [3] has shown that sidewall damage after ICP etching can be removed by suitable surface treatments.
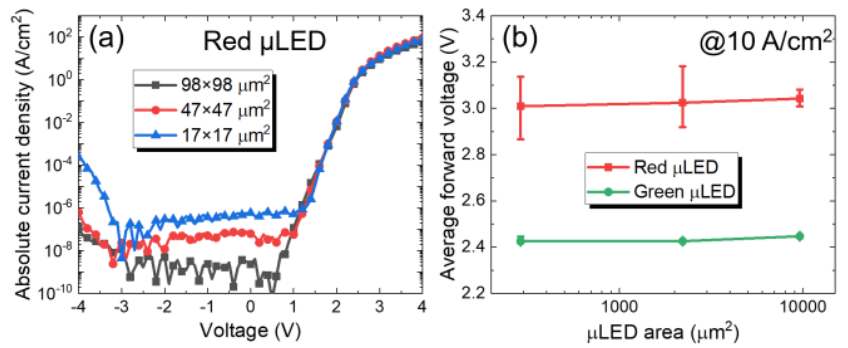

FIG. 1. (a) Absolute current density of red $\mu$ LEDs with the mesa area of $17 \times 17 \mu \mathrm{m}^{2}, 47 \times 47 \mu \mathrm{m}^{2}, 98 \times 98 \mu \mathrm{m}^{2}$ at different bias voltages. (b) Averaged forward voltages of five red and green $\mu$ LEDs at $10 \mathrm{~A} / \mathrm{cm}^{2}$.

We also compared the forward voltage of red and green $\mu$ LEDs at $10 \mathrm{~A} / \mathrm{cm}^{2}$. The average forward voltage was approximately 3.0 and $2.4 \mathrm{~V}$ for red and green $\mu$ LEDs, respectively. Generally, the red $\mu$ LEDs were expected to exhibit a lower forward voltage since the energy bandgap of the red active region was narrower. However, because we adopted $\mathrm{AlN} / \mathrm{AlGaN}$ as the barrier layers to compensate the strain in InGaN red quantum wells [23], a higher bias voltage was required for carriers to pass through the whole active region. Therefore, our red $\mu$ LEDs exhibited a higher forward voltage compared to green $\mu$ LEDs. Additionally, the average forward voltage for both red and green $\mu$ LEDs was independent of the $\mu$ LED dimension, demonstrating good current spreading and low series resistance in our $\mu$ LEDs [24].
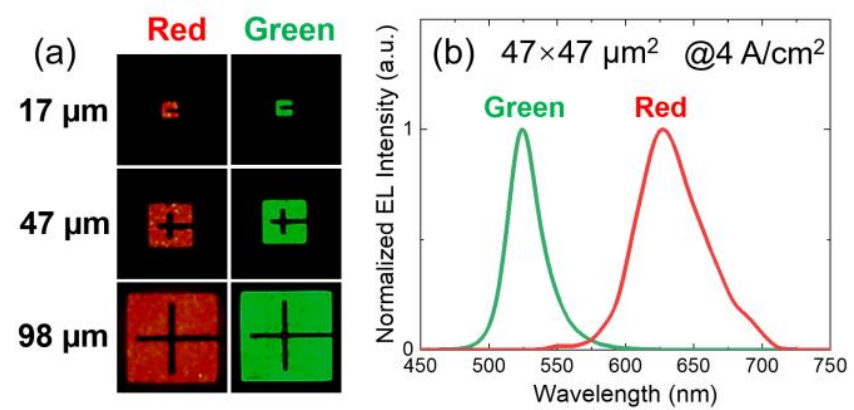

FIG. 2. (a) EL emission images of red and green $\mu$ LEDs with the mesa area of $17 \times 17 \mu \mathrm{m}^{2}, 47 \times 47 \mu \mathrm{m}^{2}, 98 \times 98 \mu^{2}$. (b) Normalized EL spectra of red and green $\mu$ LEDs with an area of $47 \times 47 \mu \mathrm{m}^{2}$. The current density was $4 \mathrm{~A} / \mathrm{cm}^{2}$.

We captured the emission patterns of red and green $\mu$ LEDs at 4 $\mathrm{A} / \mathrm{cm}^{2}$, as shown in Figure 2(a). Clearly, most device area of the red and green $\mu$ LEDs from $98 \times 98$ to $17 \times 17 \mu^{2}$ exhibited uniform luminescence although some bright spots were observed in the red $\mu$ LEDs. The common red LEDs with a large mesa area in our previous work [22] displayed many dark points in the emission patterns. These dark points were usually corresponding to the defects in the InGaN red active region. However, these dark points were not found in the red $\mu$ LEDs because of much smaller mesa areas, demonstrating high crystal quality for our red $\mu$ LEDs. Figure 2(b) shows the typical EL spectra for $47 \times 47 \mu \mathrm{m}^{2}$ red and green $\mu$ LEDs at
$4 \mathrm{~A} / \mathrm{cm}^{2}$. Two small additional peaks at around $550 \mathrm{~nm}$ and $690 \mathrm{~nm}$ were found at the tails of the spectrum of red $\mu$ LEDs. The additional peaks were regarded as the emission from localized states at low current densities and would disappear with increasing current density.

The current density dependences of the peak wavelength and FWHM were investigated from 2 to $50 \mathrm{~A} / \mathrm{cm}^{2}$ at RT. Figures 3(a) and 3(b) shows the average peak wavelength and FWHM for red and green $\mu$ LEDs with the dimension of $47 \times 47 \mu \mathrm{m}^{2}$ and $98 \times 98 \mu \mathrm{m}^{2}$. We measured three chips and calculated the average values to represent the typical results. From Figures 3(a) and 3(b), we discovered that the peak wavelength and FWHM behaviors at different current densities were independent of the dimensions of red and green $\mu$ LEDs. The similar behaviors reflected that the polarization field and the indium fluctuation in $\mathrm{InGaN}$ red or green active regions were identical despite of the shrinking of the $\mu$ LED sizes.

Red $\mu$ LEDs exhibited a much larger blue-shift (around $35 \mathrm{~nm}$ ) compared to green $\mu$ LEDs (around $6 \mathrm{~nm}$ ) at 2 to $50 \mathrm{~A} / \mathrm{cm}^{2}$. The larger blue-shift of the peak wavelength originated from the stronger QCSE existing in the red InGaN active region. The average FWHM dropped to the minimum values of $50 \mathrm{~nm}$ for red $\mu$ LEDs and $27 \mathrm{~nm}$ for green $\mu$ LEDs at low current densities, respectively. This behavior was related to the saturation of the emission from deep localized states in the InGaN-based red and green active region [25]. After the minimum value, the average FWHM of red $\mu$ LEDs increased with the current densities slightly. Our previous work [22] found that the reason for the broad FWHMs was the heat generation in devices under high direct current injection. The heat was usually generated around the defect area because defects contributed as thermal resisters [26]. However, green $\mu$ LEDs did not show significant broaden FWHMs at high current densities. We presumed that the thermal effect could be ignored due to the low defect densities in the InGaN green active region.
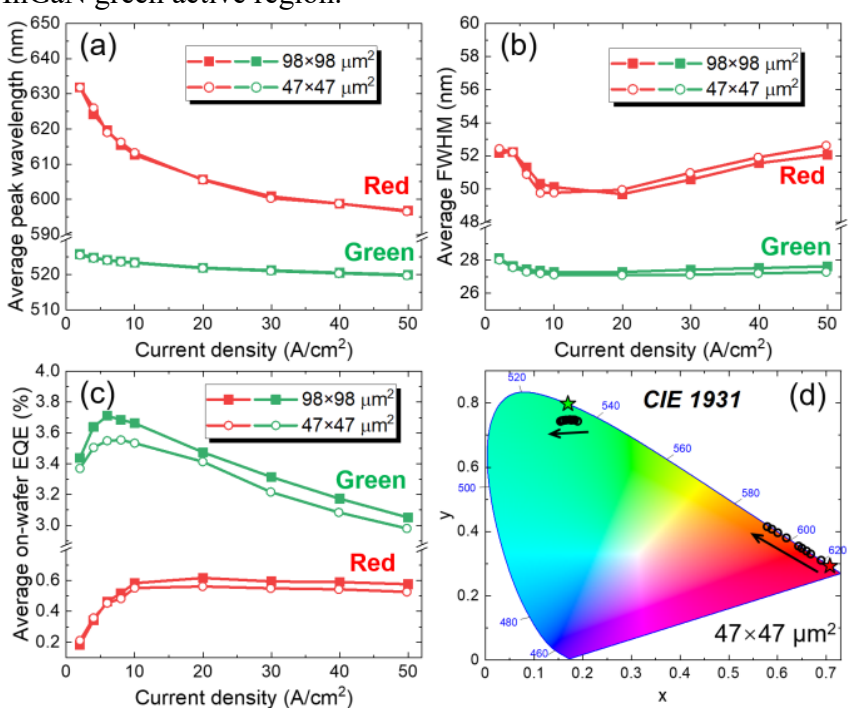

FIG. 3. (a) Average peak wavelength, (b) average FWHM, and (c) average on-wafer EQE of three red and green $\mu$ LEDs with the area of $47 \times 47 \mu \mathrm{m}^{2}, 98 \times 98 \mu \mathrm{m}^{2}$ at different current densities. (d) CIE diagram of a red and green $\mu$ LED with the area of $47 \times 47 \mu \mathrm{m}^{2}$ at different current densities. The stars are the primary red and green colors defined in Rec. 2020.

We also calculated the average on-wafer EQEs for red and green $\mu$ LEDs at different current densities from 2 to $50 \mathrm{~A} / \mathrm{cm}^{2}$, as shown in Figure 3(c). The on-wafer EQE first rose to the maximum values at the peak current density of $6 \mathrm{~A} / \mathrm{cm}^{2}$ and $8 \mathrm{~A} / \mathrm{cm}^{2}$ for $98 \times 98 \mu \mathrm{m}^{2}$ and $47 \times 47 \mu \mathrm{m}^{2}$ green $\mu \mathrm{LEDs}$, respectively. And then, it suffered from an efficiency droop and dropped quickly with the increasing current 
densities. The maximum on-wafer EQE was 3.7(1)\% and 3.5(5)\% for $98 \times 98 \mu \mathrm{m}^{2}$ and $47 \times 47 \mu \mathrm{m}^{2}$ green $\mu$ LEDs, respectively. From these data, we concluded that the peak current density would increase while the maximum on-wafer EQE would decrease for green $\mu$ LEDs when the device dimension shrank. The results were mostly caused by surface recombination at the active region edges, as explained in previous reports $[27,28]$.

The red $\mu$ LEDs behaved in a similar way at the low current densities in Figure 3(c). The average on-wafer EQE rose to the maximum values of $0.62 \%$ and $0.56 \%$ at the peak current density of $20 \mathrm{~A} / \mathrm{cm}^{2}$ for $98 \times 98 \mu \mathrm{m}^{2}$ and $47 \times 47 \mu \mathrm{m}^{2}$ red $\mu$ LEDs, respectively. A slight reduction of the maximum on-wafer $\mathrm{EQE}$ for different device dimensions was also observed in red $\mu$ LEDs. However, unlike green $\mu$ LEDs, red $\mu$ LEDs did not show an obvious efficiency droop $(<7 \%)$ at the current density up to $50 \mathrm{~A} / \mathrm{cm}^{2}$. We assumed that this phenomenon was due to higher dislocation densities in red $\mu$ LEDs than green $\mu$ LEDs [29]. The efficiency droop of our red $\mu$ LEDs was better than the previous work [14], which was assumed to be owing to better crystal quality and carrier confinement by AlN/AlGaN barriers in our red active region.

Furthermore, we measured the absolute output power of $47 \times 47$ $\mu \mathrm{m}^{2}$ green $\mu$ LEDs in the integrating sphere and determined the calibration factor for an on-wafer light output measurement vs. absolute output measurement in the integrating sphere. This calibration method was also used in other works [13,14]. Green $\mu$ LEDs in this work were not encapsulated with resin when measured in the integrating sphere. From Figures 3(a) and 3(c), the average onwafer EQE of $47 \times 47 \mu \mathrm{m}^{2}$ red $\mu$ LEDs was $0.36 \%$ at the peak wavelength of $626 \mathrm{~nm}$ at $4 \mathrm{~A} / \mathrm{cm}^{2}$. Based on the calibration factor, an absolute EQE of approximately $0.87 \%$ could be expected from our $47 \times 47 \mu \mathrm{m}^{2}$ red $\mu$ LEDs when measured in the integrating sphere. This estimated EQE value exceeds the reported one $(>0.6 \%)$ calculated by the same method in the previous work [14].

The CIE 1931 positions for red and green $\mu$ LEDs at 2 to 50 $\mathrm{A} / \mathrm{cm}^{2}$ are shown in Figure 3(d). These positions determined the physiologically perceived colors in human color vision. According to the recommendation (Rec.) 2020 standard, the primary red and green colors were located at the coordinates of $(0.708,0.292)$ and $(0.17,0.797)$, respectively. They were marked as red and green stars as reference points in Figure 3(d). Clearly, a small gap existed between the coordinates of green $\mu$ LEDs and the defined green color in Rec. 2020. This gap illustrated that the color saturation of green $\mu$ LEDs was insufficient, which was originated from broad FWHM of InGaN-based green $\mu$ LEDs. Additionally, the distance between the coordinates of green $\mu$ LEDs and the reference point of green color in Rec. 2020 remained almost constant with the current density.

However, red $\mu$ LEDs exhibited different movements relative to green $\mu$ LEDs. At the low current density, the position was close to the reference point of red color in Rec. 2020. When the current density was increased, the coordinate position shifted farther away from the reference point. As a result, the color gamut area coverage would be reduced when red $\mu$ LEDs operated at higher current densities. Since this position shift of red $\mu$ LEDs is originated from a large blue-shift of the peak wavelength, eliminating or reducing the QCSE in InGaN red active region is necessary to decrease this shift. Another approach is to fabricate longer peak wavelengths of red $\mu L E D s$, which could guarantee the gamut area coverage even at high current densities. Although the FWHM of red $\mu$ LEDs was much larger than that of green $\mu$ LEDs, the values of 50-52 nm seemed to be acceptable because all positions were located at the edge of the color space in the CIE 1931 diagram.

Finally, we investigated the temperature dependence of the EL integrated intensity and peak wavelength for the $47 \times 47 \mu \mathrm{m}^{2}$ red and green $\mu$ LEDs, as shown in Figures 4(a) and 4(b), respectively. The
EL intensities of red and green $\mu$ LEDs dropped with temperature, which is usually called the thermal droop [30]. From the fitting dot lines in Figure 4(a), the characteristic temperatures were obtained as $50 \mathrm{~K}$ and $411 \mathrm{~K}$ at $10 \mathrm{~A} / \mathrm{cm}^{2}$ for red and green $\mu$ LEDs, respectively. The smaller characteristic temperature for red $\mu$ LEDs indicated that the $\mathrm{InGaN}$ red active region has many Shockley-Read-Hall (SRH) recombination centers [30], which are related to the defect density and become more effective with temperature.

Figure 4(b) shows that the red and green $\mu$ LEDs exhibit the redshift of the peak wavelength with increasing temperature. The redshift is caused by the shrinking of the InGaN bandgap. The redshift coefficients of the peak wavelength was $0.126 \mathrm{nmK}^{-1}$ and $0.038 \mathrm{nmK}^{-1}$ for red and green $\mu L E D s$, respectively. The redshift coefficient of InGaN red $\mu$ LEDs was slightly smaller than that of AlInGaP red LEDs [31,32], indicating smaller temperature dependence of InGaN materials. However, InGaN red $\mu$ LEDs show the larger coefficient compared to green $\mu$ LEDs. We attributed this larger coefficient to more SRH recombination centers in InGaN red active region. The SRH recombination became effective and led to less carrier density for radiative recombination in the active region with temperature. As a result, the red active region suffered from more QCSE and had a larger redshift of the peak wavelength.
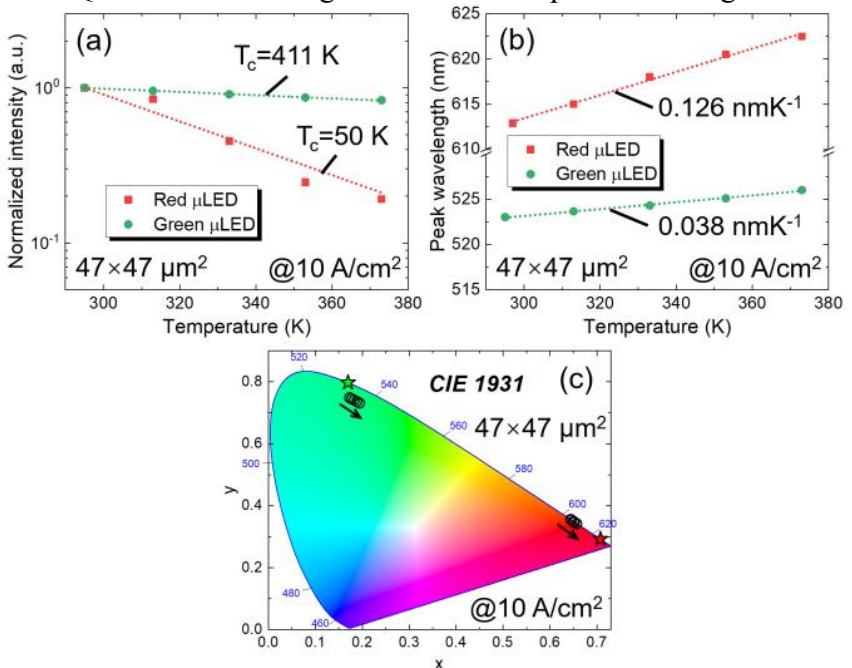

Figure 4. (a) Normalized intensity, (b) peak wavelength, and (c) CIE diagram of $47 \times 47 \mu \mathrm{m}^{2}$ red and green $\mu$ LEDs operated at different temperatures at $10 \mathrm{~A} / \mathrm{cm}^{2}$.

To estimate the influence of temperature in the color space, we plotted the temperature dependence of the color coordinates in the CIE 1931 diagram (Figure 4(c)). The movements of red and green $\mu$ LEDs are marked with arrows in Figure 4(c). The green $\mu$ LEDs moved away from the reference point of green color in Rec. 2020 (green star in Figure 4(c)) with increasing temperature. Therefore, the gamut area coverage would be reduced due to this movement of green $\mu$ LEDs. On the contrary, red $\mu$ LEDs moved towards the reference point of red color in Rec. 2020 (red star in Figure 4(c)) with temperature. Although the movement of red $\mu$ LEDs led to the hue variation, the gamut area coverage could be increased.

In summary, we investigated the performance of InGaN-based red/green $\mu$ LEDs ranging from $98 \times 98$ to $17 \times 17 \mu \mathrm{m}^{2}$. The average forward voltage at $10 \mathrm{~A} / \mathrm{cm}^{2}$ was independent of the dimension of $\mu$ LEDs. But the reverse leakage currents of red $\mu$ LEDs increased with the reduction of device dimension. A large blue-shift of the peak wavelength $(\sim 35 \mathrm{~nm})$ and a broad FWHM ( $\geq 50 \mathrm{~nm})$ at 2 to $50 \mathrm{~A} / \mathrm{cm}^{2}$ were observed in red $\mu$ LEDs. An on-wafer EQE of $0.36 \%$ (corresponding to an expected absolute EQE of $0.87 \%$ ) at the peak wavelength of $626 \mathrm{~nm}$ at $4 \mathrm{~A} / \mathrm{cm}^{2}$ was achieved from the $47 \times 47 \mu \mathrm{m}^{2}$ 
red $\mu$ LEDs. Considering the positions in the CIE 1931 diagram, we recommend suppressing the peak wavelength blue-shift for the red $\mu$ LEDs and making FWHM narrower for the green $\mu$ LEDs to improve the color quality and stability. InGaN red $\mu \mathrm{LEDs}$ suffer from a severe thermal droop compared to green $\mu$ LEDs, but the

Funding. King Abdullah University of Science and Technology (KAUST) (BAS/1/1676-01-01)

Acknowledgments. The fabrication processes in this work were supported by Nanofabrication Core Labs in KAUST.

Disclosures. The authors declare no conflicts of interest.

\section{References}

1. T. Z. Wu, C. W. Sher, Y. Lin, C. F. Lee, S. J. Liang, Y. J. Lu, S. W. H. Chen, W. J. Guo, H. C. Kuo, and Z. Chen, "Mini-LED and Micro-LED: Promising Candidates for the Next Generation Display Technology," Appl. Sci. 8, 1557 (2018).

2. M. S. Wong, S. Nakamura, and S. P. DenBaars, "Review—Progress in High Performance III-Nitride Micro-Light-Emitting Diodes," ECS J. Solid State Sci. Technol. 9, 015012 (2019)

3. M. S. Wong, C. Lee, D. J. Myers, D. Hwang, J. A. Kearns, T. Li, J. S. Speck, S. Nakamura, and S. P. DenBaars, "Size-independent peak efficiency of IIInitride micro-light-emitting-diodes using chemical treatment and sidewall passivation," Appl. Phys. Express 12, 097004 (2019).

4. J. M. Smith, R. Ley, M. S. Wong, Y. H. Baek, J. H. Kang, C. H. Kim, M. J. Gordon, S. Nakamura, J. S. Speck, and S. P. DenBaars, "Comparison of sizedependent characteristics of blue and green InGaN microLEDs down to $1 \mu \mathrm{m}$ in diameter," Appl. Phys. Lett. 116, 071102 (2020).

5. T. Tao, T. Zhi, X. Cen, B. Liu, Q. Wang, Z. L. Xie, P. Chen, D. J. Chen, Y. G. Zhou, Y. D. Zheng, and R. Zhang, "Hybrid Cyan Nitride/Red Phosphors White Light-Emitting Diodes With Micro-Hole Structures," IEEE Photon. J. 10, 1 (2018).

6. S.-W. H. Chen, Y.-M. Huang, K. J. Singh, Y.-C. Hsu, F.-J. Liou, J. Song, J. Choi, P.-T. Lee, C.-C. Lin, Z. Chen, J. Han, T. Wu, and H.-C. Kuo, "Full-color micro-LED display with high color stability using semipolar (20-21) InGaN LEDs and quantum-dot photoresist," Photonics Res. 8, 630-636 (2020).

7. G. S. Chen, B. Y. Wei, C. T. Lee, and H. Y. Lee, "Monolithic Red/Green/Blue Micro-LEDs With HBR and DBR Structures," IEEE Photon. Technol. Lett. 30, 262-265 (2018).

8. Z. Zhuang, J. Dai, B. Liu, X. Guo, Y. Li, T. Tao, T. Zhi, G. Zhang, Z. Xie, H. Ge, Y. Shi, Y. Zheng, and R. Zhang, "Improvement of color conversion and efficiency droop in hybrid light-emitting diodes utilizing an efficient nonradiative resonant energy transfer," Appl. Phys. Lett. 109, 141105 (2016).

9. M. S. Wong, J. A. Kearns, C. Lee, J. M. Smith, C. Lynsky, G. Lheureux, H. Choi, J. Kim, C. Kim, S. Nakamura, J. S. Speck, and S. P. DenBaars, "Improved performance of AlGaInP red micro-light-emitting diodes with sidewall treatments," Opt. Express 28, 5787-5793 (2020).

10. J. Bai, Y. Cai, P. Feng, P. Fletcher, C. Zhu, Y. Tian, and T. Wang, "Ultrasmall, Ultracompact and Ultrahigh Efficient InGaN Micro Light Emitting Diodes ( $\mu$ LEDs) with Narrow Spectral Line Width," ACS Nano 14, 6906-6911 (2020).

11. F. Gou, E.-L. Hsiang, G. Tan, P.-T. Chou, Y.-L. Li, Y.-F. Lan, and S.-T. $\mathrm{Wu}$, "Angular color shift of micro-LED displays," Opt. Express 27, A746A757 (2019)

12. J.-T. Oh, S.-Y. Lee, Y.-T. Moon, J. H. Moon, S. Park, K. Y. Hong, K. Y. Song, C. Oh, J.-I. Shim, H.-H. Jeong, J.-O. Song, H. Amano, and T.-Y. Seong, "Light output performance of red AlGaInP-based light emitting diodes with different chip geometries and structures," Opt. Express 26, 11194-11200 (2018).

13. A. Dussaigne, F. Barbier, B. Damilano, S. Chenot, A. Grenier, A. M. Papon, B. Samuel, B. B. Bakir, D. Vaufrey, J. C. Pillet, A. Gasse, O. Ledoux, M. Rozhavskaya, and D. Sotta, "Full InGaN red light emitting diodes," J. Appl. Phys. 128, 135704 (2020).

14. S. S. Pasayat, C. Gupta, M. S. Wong, R. Ley, M. J. Gordon, S. P. DenBaars, S. Nakamura, S. Keller, and U. K. Mishra, "Demonstration of ultra-small $(<10$ $\mu \mathrm{m}) 632 \mathrm{~nm}$ red InGaN micro-LEDs with useful on-wafer external quantum efficiency $(>0.2 \%)$ for mini-displays," Appl. Phys. Express 14, 011004 coordinates of the red $\mu$ LEDs in the CIE 1931 diagram move towards the primary red color (Rec. 2020) with temperature. This opposite movement to the green $\mu$ LEDs helps to increase the gamut area coverage in the color space.

(2020)

15. S. Zhang, J. Zhang, J. Gao, X. Wang, C. Zheng, M. Zhang, X. Wu, L. Xu, J. Ding, Z. Quan, and F. Jiang, "Efficient emission of InGaN-based lightemitting diodes: toward orange and red," Photonics Res. 8, 1671-1675 (2020). 16. K. Ohkawa, T. Watanabe, M. Sakamoto, A. Hirako, and M. Deura, "740$\mathrm{nm}$ emission from InGaN-based LEDs on c-plane sapphire substrates by MOVPE," J. Cryst. Growth 343, 13-16 (2012).

17. K. Ohkawa, F. Ichinohe, T. Watanabe, K. Nakamura, and D. Iida, "Metalorganic vapor-phase epitaxial growth simulation to realize highquality and high-In-content InGaN alloys," J. Cryst. Growth 512, 69-73 (2019).

18. D. Iida, Z. Zhuang, P. Kirilenko, M. Velazquez-Rizo, and K. Ohkawa, "Demonstration of low forward voltage InGaN-based red LEDs," Appl. Phys. Express 13, 031001 (2020).

19. D. Iida, Z. Zhuang, P. Kirilenko, M. Velazquez-Rizo, M. A. Najmi, and K. Ohkawa, "633-nm InGaN-based red LEDs grown on thick underlying GaN layers with reduced in-plane residual stress," Appl. Phys. Lett. 116, 162101 (2020).

20. D. Iida, K. Niwa, S. Kamiyama, and K. Ohkawa, "Demonstration of InGaN-based orange LEDs with hybrid multiple-quantum-wells structure," Appl. Phys. Express 9, 111003 (2016).

21. Z. Zhuang, D. Iida, P. Kirilenko, M. Velazquez-Rizo, and K. Ohkawa, "Optimal ITO transparent conductive layers for InGaN-based amber/red light-emitting diodes," Opt. Express 28, 12311-12321 (2020).

22. Z. Zhuang, D. Iida, and K. Ohkawa, "Effects of size on the electrical and optical properties of InGaN-based red light-emitting diodes," Appl. Phys. Lett. 116, $173501(2020)$

23. D. Iida, S. Lu, S. Hirahara, K. Niwa, S. Kamiyama, and K. Ohkawa, "Enhanced light output power of InGaN-based amber LEDs by straincompensating AlN/AlGaN barriers," J. Cryst. Growth 448, 105-108 (2016). 24. P. Li, H. Zhang, H. Li, M. Iza, Y. Yao, M. S. Wong, N. Palmquist, J. S. Speck, S. Nakamura, and S. P. DenBaars, "Size-independent low voltage of InGaN micro-light-emitting diodes with epitaxial tunnel junctions using selective area growth by metalorganic chemical vapor deposition," Opt. Express 28, 18707-18712 (2020).

25. H. Wang, Z. Ji, S. Qu, G. Wang, Y. Jiang, B. Liu, X. Xu, and H. Mino, "Influence of excitation power and temperature on photoluminescence in InGaN/GaN multiple quantum wells," Opt. Express 20, 3932-3940 (2012). 26. S. Okamoto, N. Saito, K. Ito, B. Ma, K. Morita, D. Iida, K. Ohkawa, and Y. Ishitani, "Energy transport analysis in a Ga0.84In $0.16 \mathrm{~N} / \mathrm{GaN}$ heterostructure using microscopic Raman images employing simultaneous coaxial irradiation of two lasers," Appl. Phys. Lett. 116, 142107 (2020).

27. S. S. Konoplev, K. A. Bulashevich, and S. Y. Karpov, "From Large-Size to Micro-LEDs: Scaling Trends Revealed by Modeling," Phys. Status Solid A 215, 1700508 (2018).

28. P. F. Tian, J. J. D. McKendry, Z. Gong, B. Guilhabert, I. M. Watson, E. D. Gu, Z. Z. Chen, G. Y. Zhang, and M. D. Dawson, "Size-dependent efficiency and efficiency droop of blue InGaN micro-light emitting diodes," Appl. Phys. Lett. 101, 4 (2012).

29. M. F. Schubert, S. Chhajed, J. K. Kim, E. F. Schubert, D. D. Koleske, M. H. Crawford, S. R. Lee, A. J. Fischer, G. Thaler, and M. A. Banas, "Effect of dislocation density on efficiency droop in GaInN/GaN light-emitting diodes," Appl. Phys. Lett. 91, 231114 (2007).

30. D. S. Meyaard, Q. Shan, J. Cho, E. F. Schubert, S.-H. Han, M.-H. Kim, C. Sone, S. J. Oh, and J. K. Kim, "Temperature dependent efficiency droop in GaInN light-emitting diodes with different current densities," Appl. Phys. Lett. 100, 081106 (2012)

31. H. K. Lee, D. H. Lee, Y. M. Song, Y. T. Lee, and J. S. Yu, "Thermal measurements and analysis of AlGaInP/GaInP MQW red LEDs with different chip sizes and substrate thicknesses," Solid-State Electron. 56, 79-84 (2011). 32. M. S. Kim, H. K. Lee, and J. S. Yu, "Device characteristics and thermal analysis of AlGaInP-based red monolithic light-emitting diode arrays," Semicond. Sci. Technol. 28, 025005 (2013). 